\title{
Research Article \\ Finding of principle square root of a real number by using interpolation method
}

\author{
Rajendra Prasad Regmi
}

Lecturer, Department of Mathematics, P.N. Campus, Pokhara

Email: rajendraprasadregmi@yahoo.com

Article History

Received 17 June, 2018

Revised 27 July 2018

Accepted 30 November 2018

\section{Abstract}

There are various methods of finding the square roots of positive real number. This paper deals with finding the principle square root of positive real numbers by using Lagrange's and Newton's interpolation method. The interpolation method is the process of finding the values of unknown quantity (y) between two known quantities.

Keywords: Interpolation, real number, square root

(C) 2018 The Author. Published by JRCC, Janapriya Multiple Campus

ISSN 2362-1516

\section{Introduction}

The square root of a number is value that when multiplied by itself, gives the number. All positive real number has two square roots, one positive square root and one negative square root. The positive square root is sometimes referred to as the principle square root. A square root is written with a radical symbol $\sqrt{ }$ and the number or expression inside the radical symbol, below denoted a called radicand $\sqrt{a}$. Negative numbers don't have real square roots since a square is either positive or 0 . If the square root of an integer is another integer then the square is called

perfect square. For example: 36 . It is perfect square

$$
\sqrt{36}= \pm 6
$$

If the radicant is not perfect square i.e the square root is not a whole number then we have to approximate the square root

$$
\pm \sqrt{3}= \pm 1.73205 \approx \pm 1.7
$$


The square roots of numbers that are not a perfect square are members of the irrational numbers.

The process of finding the value of ${ }^{y\left(x_{i}\right)}$ for corresponding value of $x_{i} \in\left(x_{0}, x_{n}\right)$ is called interpolation.

The process of finding the value of ${ }^{y\left(x_{i}\right)}$ for corresponding value of $x_{i} \notin\left(x_{0}, x_{n}\right)$ is called extrapolation.

To obtain the value of unknown quality by using Newton's interpolation method we use three types of formula namely, forward difference, backward differences and central difference. Forward difference: The first order forward difference of $f(x)$ is the change in $f(x)$ when $x$ is increased by a positive difference $h$. The operation is carried out through the notation $\Delta$ Backward difference: The first order backward difference of $f(x)$ is the change in $f(x)$ when $x$ decreases by a positive difference $h$. The operation is carried out through the notation $\nabla$ Central difference: The forward and backward differences are mainly useful in interpolating the values near the beginning and the end of the table respectively. Central differences are particularly useful in interpolating for value of $\mathrm{x}$ in the interior of the table.

For equal interval we use the following method to find the interpolation:

1. Newton's forward difference interpolation formula.

2. Newton's backward difference interpolation formula.

For unequal interval we use the following methods to find the interpolation:

1. Newton's divided difference formula. 2. Stiring central difference formula.

3. Gauss central difference formula. 4. Bessel's interpolation formula.

5. Lagring's interpolation formula

Table 1

Newton's Divided Difference Table

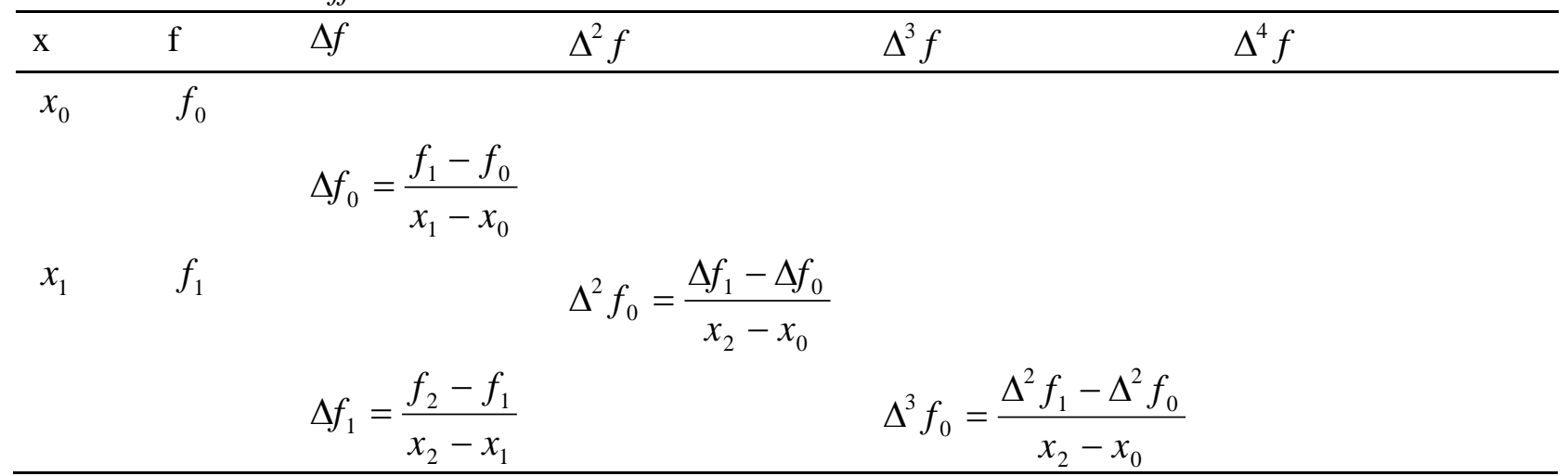




\begin{tabular}{lll}
\hline$x_{2} \quad f_{2}$ & $\Delta^{2} f_{1}=\frac{\Delta f_{2}-\Delta f_{1}}{x_{3}-x_{1}}$ & $\Delta^{3} f_{1}=\frac{\Delta^{2} f_{2}-\Delta^{2} f_{1}}{x_{3}-x_{1}}$ \\
$x_{3} \quad f_{3}=\frac{f_{3}-f_{2}}{x_{3}-x_{2}}$ & $\Delta^{2} f_{2}=\frac{\Delta f_{3}-\Delta f_{2}}{x_{4}-x_{2}}$ \\
$x_{4}$ & $f_{4}=\frac{f_{4}-f_{3}}{x_{4}-x_{3}}$
\end{tabular}

Table 2

Newton's Forward Difference Table

\begin{tabular}{|c|c|c|c|c|c|}
\hline $\mathrm{x}$ & $\mathrm{y}$ & $\Delta y$ & $\Delta^{2} y$ & $\Delta^{3} y$ & $\Delta^{4} y$ \\
\hline \multirow[t]{2}{*}{$x_{0}$} & $y_{0}$ & & & & \multirow{7}{*}{$\Delta^{4} y_{0}=\Delta^{3} y_{1}-\Delta^{3} y_{0}$} \\
\hline & & $\Delta y_{0}=y_{1}-y_{0}$ & & & \\
\hline \multirow[t]{2}{*}{$x_{1}$} & $y_{1}$ & & $\Delta^{2} y_{0}=\Delta y_{1}-\Delta y_{0}$ & & \\
\hline & & $\Delta y_{1}=y_{2}-y_{1}$ & & $\Delta^{3} y_{0}=\Delta^{2} y_{1}-\Delta^{2} y_{0}$ & \\
\hline$x_{2}$ & $y_{2}$ & & $\Delta^{2} y_{1}=\Delta y_{2}-\Delta y_{1}$ & & \\
\hline \multirow[t]{2}{*}{$x_{3}$} & $y_{3}$ & $\Delta y_{2}=y_{3}-y_{2}$ & $\Delta^{2} y_{2}=\Delta y_{2}-\Delta y_{1}$ & $\Delta^{3} y_{1}=\Delta^{2} y_{2}-\Delta^{2} y_{1}$ & \\
\hline & & $\Delta y_{3}=y_{4}-y_{3}$ & & & \\
\hline$x_{4}$ & $y_{4}$ & & & & \\
\hline
\end{tabular}

Table 3

Newton's Backward Difference Table

\begin{tabular}{|c|c|c|c|c|c|}
\hline $\mathrm{x}$ & $\mathrm{y}$ & $\nabla y$ & $\nabla^{2} y$ & $\nabla^{3} y$ & $\nabla^{4} y$ \\
\hline \multirow[t]{2}{*}{$x_{0}$} & $y_{0}$ & & & & \\
\hline & & $\nabla y_{0}=y_{1}-y_{0}$ & & & \\
\hline$x_{1}$ & $y_{1}$ & & $\nabla^{2} y_{0}=\nabla y_{1}-\nabla y_{0}$ & & \\
\hline & & $\nabla y_{1}=y_{2}-y_{1}$ & & $\nabla^{3} y_{0}=\nabla^{2} y_{1}-\nabla^{2} y_{0}$ & \\
\hline$x_{2}$ & $y_{2}$ & & $\nabla^{2} y_{1}=\nabla y_{2}-\nabla y_{1}$ & & $\nabla^{4} y_{0}=\nabla^{3} y_{1}-\nabla^{3} y_{0}$ \\
\hline & & $\nabla y_{2}=y_{3}-y_{2}$ & & $\nabla^{3} y_{1}=\nabla^{2} y_{2}-\nabla^{2} y_{1}$ & \\
\hline$x_{3}$ & $y_{3}$ & & $\nabla^{2} y_{2}=\nabla y_{2}-\nabla y_{1}$ & & \\
\hline
\end{tabular}




$$
\nabla y_{3}=y_{4}-y_{3}
$$

\begin{tabular}{ll}
$x_{4}$ & $y_{4}$ \\
\hline
\end{tabular}

\section{Result and Discussion}

To find the principle square root of real number by interpolation method it is necessary to know the Lagrange's interpolation formula as well as Newton's interpolation formula.

\section{Lagrange's interpolation formula}

Suppose $\mathrm{y}=\mathrm{f}(\mathrm{x})$ be function with $f\left(x_{0}\right), f\left(x_{1}\right), f\left(x_{2}\right) \ldots \ldots \ldots \ldots \ldots f\left(x_{n}\right)$ corresponding to the values $x_{0}, x_{1}, x_{2} \ldots \ldots \ldots \ldots \ldots x_{n} y_{\text {then }} y_{0}=f\left(x_{0}\right), y_{1}=f\left(x_{1}\right)$, $y_{2}=f\left(x_{2}\right) \ldots \ldots \ldots \ldots \ldots y_{n}=f\left(x_{n}\right)$. then Lagrange's interpolation formula is given by $y=f(x)=\frac{\left(x-x_{1}\right)\left(x-x_{2}\right) \ldots \ldots \ldots\left(x-x_{n}\right)}{\left(x_{0}-x_{1}\right)\left(x_{0}-x_{2}\right) \ldots \ldots .\left(x_{0}-x_{n}\right)} \times y_{0} \frac{\left(x-x_{0}\right)\left(x-x_{2}\right) \ldots \ldots \ldots\left(x-x_{n}\right)}{\left(x_{1}-x_{0}\right)\left(x_{1}-x_{2}\right) \ldots \ldots .\left(x_{1}-x_{n}\right)} \times y_{1}$

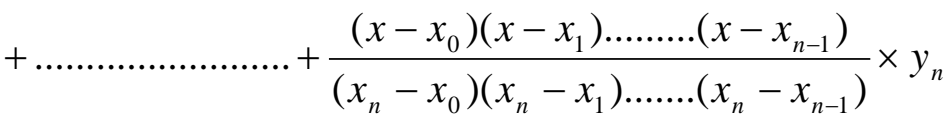

\section{Newton's divided difference formula}

Suppose $\mathrm{y}=\mathrm{f}(\mathrm{x})$ be function with $f\left(x_{0}\right), f\left(x_{1}\right), f\left(x_{2}\right) \ldots \ldots \ldots \ldots \ldots f\left(x_{n}\right)$ corresponding to the values $x_{0}, x_{1}, x_{2} \ldots \ldots \ldots \ldots \ldots x_{n} y_{\text {then }} y_{0}=f\left(x_{0}\right) \quad y_{1}=f\left(x_{1}\right)$, $y_{2}=f\left(x_{2}\right) \ldots \ldots \ldots \ldots \ldots . . . y_{n}=f\left(x_{n}\right)$. then Newton's divided difference formula is given by $y=y_{0}+\left(x-x_{0}\right) \Delta f_{0}+\left(x-x_{0}\right)\left(x-x_{1}\right) \Delta^{2} f_{0}+\ldots \ldots \ldots \ldots \ldots \ldots+\left(x-x_{0}\right)\left(x-x_{1}\right) \ldots \ldots \ldots \ldots \ldots \ldots \ldots \ldots\left(x-x_{n}\right) \Delta^{n} f_{0}$

\section{Newton's forward difference formula}

$y_{p}=y_{0}+p \Delta y_{0}+\frac{p(p-1)}{2 !} \Delta^{2} y_{0}+\frac{p(p-1)(p-2)}{3 !} \Delta^{3} y_{0}+\frac{p(p-1)(p-2)(p-3)}{4 !} \Delta^{4} y_{0}+\ldots \ldots \ldots$

Where $p=\frac{x_{p}-x_{0}}{h}$ 
$x_{p}=$ value of which interpolation is to be found

$x_{0}=$ initial value related to $x_{p}$

$\mathrm{h}=$ interval of $\mathrm{x}$.

\section{Newton's back ward difference formula}

$y_{p}=y_{n}+p \nabla y_{0}+\frac{p(p+1)}{2 !} \nabla^{2} y_{n}+\frac{p(p+1)(p+2)}{3 !} \nabla^{3} y_{n}+\frac{p(p+1)(p+2)(p+3)}{4 !} \nabla^{4} y_{n}+\ldots \ldots \ldots$

Where $p=\frac{x_{p}-x_{n}}{h}$

$x_{p}=$ value of which interpolation is to be found

$x_{n}=$ finall value related to $x_{p}$

$\mathrm{h}=$ interval of $\mathrm{x}$.

Example: Given that

\begin{tabular}{llllll}
\hline $\mathrm{X}$ & 1 & 4 & 9 & 16 & 25 \\
$\mathrm{y}$ & 1 & 2 & 3 & 4 & 5 \\
\hline
\end{tabular}

Calculate the approximate principle square root of 10.56 by using Lagrange's interpolation formula.

Here, $x_{0}=1, x_{1}=4, x_{2}=9, x_{3}=16, x_{4}=25$

Here, $y_{0}=1, y_{1}=2, y_{2}=3, y_{3}=4, y_{4}=5$

$X=10.56$

Lagrange's interpolation formula is

$$
\begin{aligned}
& y=f(x)=\frac{\left(x-x_{1}\right)\left(x-x_{2}\right) \ldots \ldots \ldots\left(x-x_{n}\right)}{\left(x_{0}-x_{1}\right)\left(x_{0}-x_{2}\right) \ldots \ldots .\left(x_{0}-x_{n}\right)} \times y_{0+} \frac{\left(x-x_{0}\right)\left(x-x_{2}\right) \ldots \ldots \ldots\left(x-x_{n}\right)}{\left(x_{1}-x_{0}\right)\left(x_{1}-x_{2}\right) \ldots \ldots .\left(x_{1}-x_{n}\right)} \times y_{1} \\
& +\ldots \ldots \ldots \ldots \ldots \ldots \ldots+\frac{\left(x-x_{0}\right)\left(x-x_{1}\right) \ldots \ldots \ldots\left(x-x_{n-1}\right)}{\left(x_{n}-x_{0}\right)\left(x_{n}-x_{1}\right) \ldots \ldots . .\left(x_{n}-x_{n-1}\right)} \times y_{n}
\end{aligned}
$$




$$
\begin{aligned}
& \text { or, } y=f(10.56)=\frac{\left(x-x_{1}\right)\left(x-x_{2}\right)\left(x-x_{3}\right)\left(x-x_{4}\right)}{\left(x_{0}-x_{1}\right)\left(x_{0}-x_{2}\right)\left(x_{0}-x_{3}\right)\left(x_{0}-x_{4}\right)} \times y_{0}+ \\
& \frac{\left(x-x_{0}\right)\left(x-x_{2}\right)\left(x-x_{3}\right)\left(x-x_{4}\right)}{\left(x_{1}-x_{0}\right)\left(x_{1}-x_{2}\right)\left(x_{1}-x_{3}\right)\left(x_{1}-x_{4}\right)} \times y_{1}+\frac{\left(x-x_{0}\right)\left(x-x_{1}\right)\left(x-x_{3}\right)\left(x-x_{4}\right)}{\left(x_{2}-x_{0}\right)\left(x_{2}-x_{1}\right)\left(x_{2}-x_{3}\right)\left(x_{2}-x_{4}\right)} \times y_{2} \\
& +\frac{\left(x-x_{0}\right)\left(x-x_{1}\right)\left(x-x_{2}\right)\left(x-x_{4}\right)}{\left(x_{3}-x_{0}\right)\left(x_{3}-x_{1}\right)\left(x_{3}-x_{2}\right)\left(x_{3}-x_{4}\right)} \times y_{3} \\
& =\frac{(10.56-4)(10.56-9)(10.56-16)(10.56-25)}{(1-4)(1-9)(1-16)(1-25)} \times 1 \\
& +\frac{(10.56-1)(10.56-9)(10.56-16)(10.56-25)}{(4-1)(4-9)(-16)(9-25)} \times 2 \\
& =3.23 . \\
& +\frac{(10.56-1)(10.56-4)(10.56-16)(10.56-25)}{(9-1)(9-4)(9-16)(9-25)} \times 3 \\
& +\frac{(10.56-1)(10.56-4)(10.56-9)(10.56-25)}{(16-1)(16-4)(16-9)(16-25)} \times 4 \\
& +0.61985+3.298+0.49831
\end{aligned}
$$

\begin{tabular}{|c|c|c|c|c|c|}
\hline $\mathrm{x}$ & $\mathrm{y}$ & $\Delta y$ & $\Delta^{2} y$ & $\Delta^{3} y$ & $\Delta^{4} y$ \\
\hline$x_{0}$ & $y_{0}$ & & & & \\
\hline \multicolumn{6}{|c|}{$\Delta y_{0}=y_{1}-y_{0}$} \\
\hline
\end{tabular}

Therefore the approximate principle square root of 10.56 is 3.23.

Example: Given that

\begin{tabular}{llllll}
\hline $\mathrm{X}$ & 7 & 10 & 13 & 16 & 19 \\
$\mathrm{y}$ & 2.64 & 3.16 & 3.60 & 4 & 4.35 \\
\hline
\end{tabular}

Calculate the approximate principle square root of 10.56 by using Newton's forward difference formula.

Table 4

Newton's Forward Difference Formula is 


\begin{tabular}{|c|c|c|c|c|c|}
\hline \multirow[t]{2}{*}{$x_{1}$} & $y_{1}$ & & $\Delta^{2} y_{0}=\Delta y_{1}-\Delta y_{0}$ & & \multirow[b]{3}{*}{$\Delta^{4} y_{0}=\Delta^{3} y_{1}-\Delta^{3} y_{0}$} \\
\hline & & $\Delta y_{1}=y_{2}-y_{1}$ & & $\Delta^{3} y_{0}=\Delta^{2} y_{1}-\Delta^{2} y_{0}$ & \\
\hline \multirow[t]{2}{*}{$x_{2}$} & $y_{2}$ & & $\Delta^{2} y_{1}=\Delta y_{2}-\Delta y_{1}$ & & \\
\hline & & $\Delta y_{2}=y_{3}-y_{2}$ & & $\Delta^{3} y_{1}=\Delta^{2} y_{2}-\Delta^{2} y_{1}$ & \\
\hline \multirow[t]{2}{*}{$x_{3}$} & $y_{3}$ & & $\Delta^{2} y_{2}=\Delta y_{2}-\Delta y_{1}$ & & \\
\hline & & $\Delta y_{3}=y_{4}-y_{3}$ & & & \\
\hline$x_{4}$ & $y_{4}$ & & & & \\
\hline
\end{tabular}

Or.

\begin{tabular}{llllll}
\hline $\mathrm{x}$ & $\mathrm{y}$ & $\Delta y$ & $\Delta^{2} y$ & $\Delta^{3} y$ & $\Delta^{4} y$ \\
\hline 7 & 2.64 & & & & \\
10 & 3.16 & $3.16-2.64=0.52$ & & $0.44-0.52=-0.08$ & \\
& & $3.60-3.16=0.44$ & & $-0.04+0.08=0.04$ & \\
13 & 3.60 & & $0.4-0.44=-0.04$ & & $-0.01-0.04=-0.05$ \\
16 & 4 & $4-3.60=0.4$ & $0.35-0.4=-0.05$ & & \\
19 & 4.35 & & & & \\
\hline
\end{tabular}

$x_{p}=10.56$ lies between 10 and 13

$x_{0}=10, y_{0}=3.16$

$\mathrm{h}=3$

$p=\frac{x_{p}-x_{0}}{h}=\frac{10.56-10}{3}=0.18666$

$y_{p}$ is value of $\mathrm{x}$ when $\mathrm{x}=10.56$

Newton's forward difference formula is

$$
y_{p}=y_{0}+p \Delta y_{0}+\frac{p(p-1)}{2 !} \Delta^{2} y_{0}+\frac{p(p-1)(p-2)}{3 !} \Delta^{3} y_{0}+\frac{p(p-1)(p-2)(p-3)}{4 !} \Delta^{4} y_{0}+\ldots \ldots \ldots
$$

